\title{
Transthoracic drainage of large Streptococcus milleri liver abscess
}

Arul Bala, MBBS, Pankaj Saxena, MCh, DNB, and Igor E. Konstantinov, MD, PhD, Perth, Australia

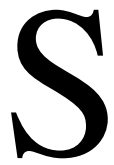

treptococcus milleri rarely causes a life-threatening infection. However, in patients with chronic disease $S$ milleri may produce suppurative infection, including liver abscess. The latter is associated with up to $100 \%$ mortality if untreated and $50 \%$ to $60 \%$ mortality after treatment. ${ }^{1}$ Although $S$ milleri seems to be the most common cause of pyogenic liver abscess, ${ }^{2}$ only a few cases are described in the medical literature.

\section{Clinical Summary}

A 45-year-old malnourished man with a history of intravenous drug use, iron deficiency anemia, and hepatitis $\mathrm{C}$ presented with 2 weeks of increasing dyspnea, fever, and malaise. Blood cultures were positive for $S$ milleri, sensitive to penicillin, erythromycin, vancomycin, tetracycline, and cephalexin. Echocardiogram was unremarkable but, incidentally, identified an intrahepatic mass adjacent to the inferior vena cava. Abdominal computed tomographic scan demonstrated a $13 \times 11 \times 8 \mathrm{~cm}$ intrahepatic abscess with extension into the subphrenic compartment (Figure 1). There



was a further extension of the abscess through the diaphragm, which was contained by parietal pleura (Figure $1, b$ to $d$ ). The abscess was adjacent to the inferior vena cava and hepatic vein (Figure 1, $d$ ). Ultrasoundguided percutaneous needle aspiration failed to drain the abscess. A large pleural effusion (Figure 2, $a$ and $b$ ) and respiratory distress despite aggressive antibiotic treatment developed in the patient, and he was transferred to our unit.

A right muscle-sparing posterolateral thoracotomy was performed through the seventh intercostal space, and $1800 \mathrm{~mL}$ of cloudy yellow effusion was drained. The lower lobe was dissected from the diaphragm, and the supradiaphragmatic collection was drained. The diaphragm and the cupola of liver were opened, and $800 \mathrm{~mL}$ of pus was drained. Five $28 \mathrm{~F}$ chest tubes were placed in the apex, oblique fissure, costophrenic recess around the hemidiaphragm, subdiaphragmatic space, and abscess cavity (Figure 2, c and $d$ ). The abscess cavity was partially obliterated with interrupted 2-0 nylon stitches over the chest tube. The patient made an uneventful recovery with complete lung reexpansion and was discharged on day 11 with a course of antibiotics (amoxicillin $1 \mathrm{~g}$ by mouth 3 times per day, teicoplanin $400 \mathrm{mg}$ intravenously once per day). Gram-positive cocci were seen on microscopy of the abscess content.

\section{Discussion}

Pyogenic liver abscesses constitute $18 \%$ of all liver abscesses with the remainder being amoebic. ${ }^{3} S$ milleri is a facultative anaerobic gram-positive coccus and has been purported to be the most common cause of pyogenic liver abscess. ${ }^{2}$ However, $S$ milleri infection per se is rarely associated with a liver abscess. Thus, Molina and colleagues, ${ }^{4}$ in a study of 51 infections of $S$ milleri in

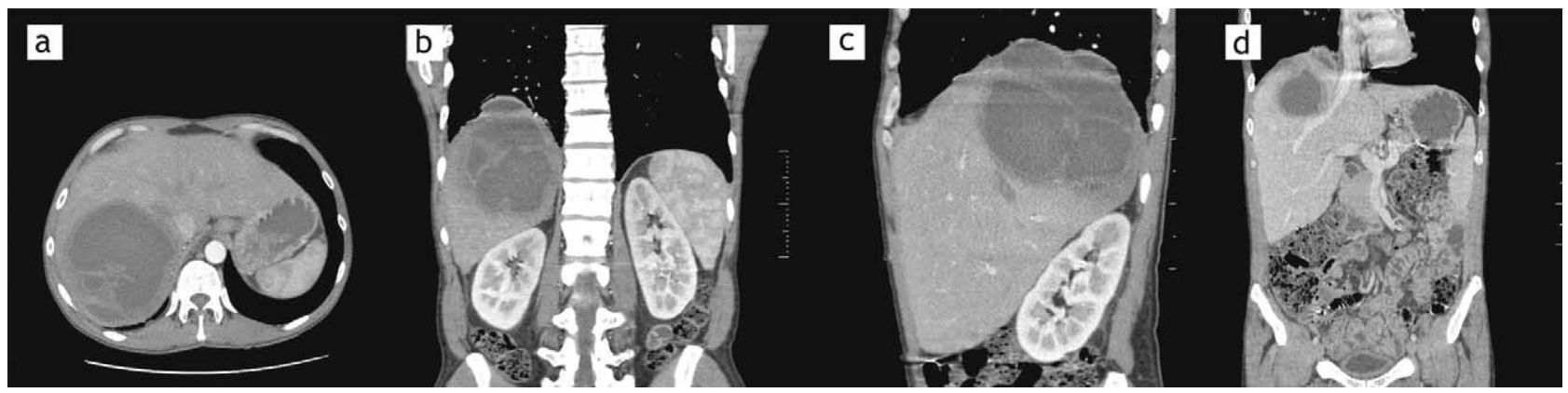

Figure 1. Computed tomographic scan demonstrated a liver abscess with multiple loculations with an extension into subdiaphragmatic space and supradiaphragmatic loculation $(a, b$, and $c)$ adjacent to the inferior vena cava and hepatic vein (d). 


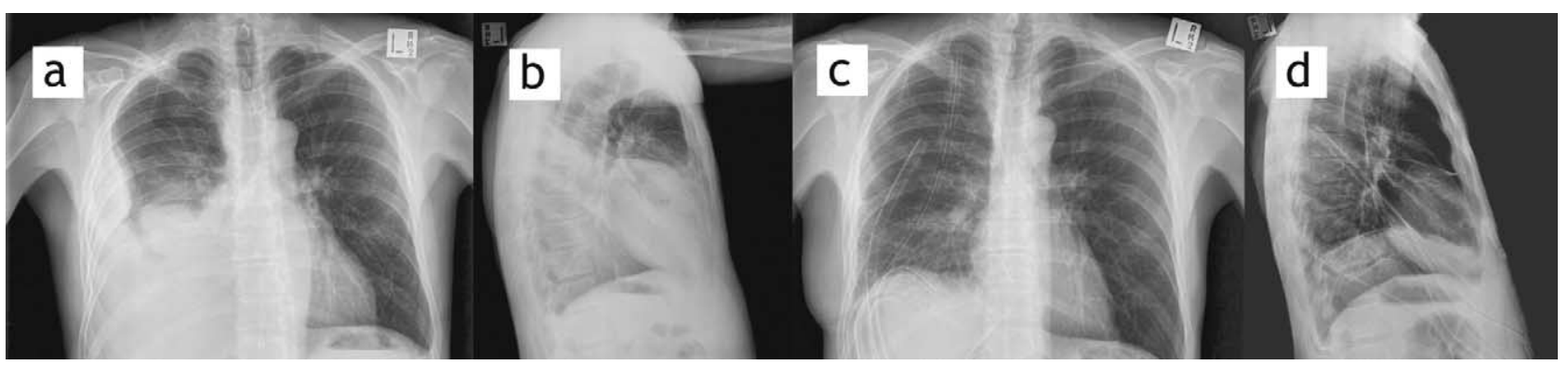

Figure 2. Chest x-ray films before (a and b) and after (c and d) surgery.

43 patients over a 2-year period, found only 2 patients with liver abscesses and 8 patients with pleural empyema. Significant underlying disease was seen in $33(73 \%)$ of these patients, including immune deficiency and gastrointestinal disease. The presumed source of infection, being the oral cavity or gastrointestinal tract, was identified in $47 \%$. S milleri alone was isolated in 19 cases (44\%), whereas $S$ milleri was mixed with anaerobes in 8 cases and enterobacteria in 13 cases. Sixteen patients (37\%) had bacteremia, and $63 \%$ required surgical drainage.

Chua and colleagues ${ }^{5}$ provide the most comprehensive literature review of $S$ milleri liver abscess to date. Of 33 cases found in their literature search, 15 were presented with clinical information and treatment results. Nine cases $(60 \%)$ had underlying chronic disease. Both studies demonstrated male preponderance. ${ }^{4,5}$

In our case, the large hepatic abscess ruptured though the hepatic parenchyma into the subdiaphragmatic space and further through the diaphragm where it was contained by parietal pleura and the lung adherent to the diaphragm. Although percutaneous drainage of a nonloculated liver abscess has been described ${ }^{6}$ and attempted in our patient, thick pus and multiple loculations rendered percutaneous drainage impossible.
In summary, although $S$ milleri infection is rare, it may become life-threatening, necessitating aggressive surgical management. Thoracotomy through the seventh intercostal space provided an excellent exposure of the diaphragm and the cavity of the hepatic abscess, while permitting washout and drainage of the pleural cavity and mediastinum.

\section{References}

1. Balasegaram M. Management of hepatic abscess. Curr Probl Surg. 1981;18:285-340.

2. Moore-Gillon JC, Eykyn SJ, Phillips I. Microbiology of pyogenic liver abscess. Br Med J. 1981;283:819-21.

3. Lodhi S, Sarwari AR, Muzammil M, Salam A, Smego RA. Features distinguishing amoebic from pyogenic liver abscess: a review of 577 adult cases. Trop Med Health. 2004;9:718-23.

4. Molina J, Leport C, Bure A, Wolff M, Michon C, Vilde J. Clinical and bacterial features of infections caused by streptococcus milleri. Scand J Infect Dis. 1991;23:659-66.

5. Chua D, Reinhart H, Sobel J. Liver abscess caused by streptococcus milleri. Rev Infect Dis. 1989;2:197-202.

6. Risi GF. Additional case of liver abscess due to streptococcus milleri. Rev Infect Dis. 1989;2:837-8. 